\title{
On Point-Sets That Support Planar Graphs ${ }^{\star}$
}

\author{
Vida Dujmovic ${ }^{1}$, William Evans ${ }^{2}$, Sylvain Lazard $^{3}$, William Lenhart ${ }^{4}$, \\ Giuseppe Liotta ${ }^{5}$, David Rappaport ${ }^{6}$, and Stephen Wismath ${ }^{7}$ \\ 1 Carleton University, Canada \\ 2 University of British Columbia, Canada \\ 3 INRIA Nancy, LORIA, France \\ 4 Williams University, U.S.A. \\ 5 Universitá degli Studi di Perugua, Italy \\ 6 Queen's University, Canada \\ 7 University of Lethbridge, Canada
}

\begin{abstract}
A universal point-set supports a crossing-free drawing of any planar graph. For a planar graph with $n$ vertices, if bends on edges of the drawing are permitted, universal point-sets of size $n$ are known, but only if the bend-points are in arbitrary positions. If the locations of the bend-points must also be specified as part of the point-set, we prove that any planar graph with $n$ vertices can be drawn on a universal set $\mathcal{S}$ of $O\left(n^{2} / \log n\right)$ points with at most one bend per edge and with the vertices and the bend points in $\mathcal{S}$. If two bends per edge are allowed, we show that $O(n \log n)$ points are sufficient, and if three bends per edge are allowed, $\Theta(n)$ points are sufficient. When no bends on edges are permitted, no universal point-set of size $o\left(n^{2}\right)$ is known for the class of planar graphs. We show that a set of $n$ points in balanced biconvex position supports the class of maximum degree 3 series-parallel lattices.
\end{abstract}

\section{Introduction}

A set of points supports the drawing of a graph $G$ if there is a one-to-one mapping $f$ of the vertices of $G$ to the points so that for all pairs of edges $(a, b),(c, d)$ in $G$ (where $a, b, c, d$ are distinct), segments $\overline{f(a) f(b)}$ and $\overline{f(c) f(d)}$ do not intersect. A set of points that supports the drawing of all $n$-vertex graphs in some class is called universal for that class, or simply universal if the class is all planar graphs. The size of any universal point-set for planar graphs requires at least $1.235 n$ points as shown by Kurowski [10] (see also Chrobak and Karloff [3]). Early graph drawing results, such as the canonical ordering technique of de Frasseix, Pach, Pollack [4] and Schnyder's embedding [11] demonstrate that an $n \times n$ grid of points is a universal point-set. However, no universal point-set of size $o\left(n^{2}\right)$ is known.

* This paper was initiated at the 2011 McGill/INRIA/UVictoria Bellairs workshop. Discussion with other participants is gratefully acknowledged. Research supported by NSERC, and by MIUR of Italy under project AlgoDEEP prot. 2008TFBWL4. 
Smaller universal point-sets for sub-classes of planar graphs are known. For example, any outerplanar graph can be drawn on any set of $n$ points in general position [8. Indeed, if the point-set is in convex position, then it supports exactly the family of outerplanar graphs. Determining other families of planar graphs for which universal point-sets of size $n$ exist is an interesting problem. We examine a particular type of point-set, of size $n$, in which points are arranged in biconvex position, and show that it supports the drawing of all maximum degree 3 series-parallel lattices, a class of graphs that contains members that are not outerplanar. These notions are precisely defined in Section 2 ,

The main contributions in this paper are stated in Theorems 1 and 2, and pertain to universal point-sets for straight-line drawings, and drawings with bends respectively.

Theorem 1. For all $n$, there exist universal point-sets of cardinality $n$ that support the family of maximum degree 3 series-parallel lattices with $n$ vertices.

Suppose we relax the definition of support to allow edges of the graph to map to polylines composed of (at most) $k+1$ line segments. In other words, we allow edges that "bend" at most $k$ times. In this case, universal point-sets of size $n$ exist for two bends [9] and even one bend [7. However, these results assume that the bend-points can be placed in arbitrary locations and these bend-points are not included as part of the universal point-set. It is natural to ask if there exists a point-set that supports all planar graphs where each vertex and each bend-point occurs at a point in the set. As before, we require all pairs of edges $(a, b)$ and $(c, d)$ (where $a, b, c, d$ are distinct) to map to non-intersecting polylines. Previous to this paper, no such point-set of cardinality $o\left(n^{2}\right)$ was known for any value of $k$. Extending the results of [7] and [9] in a straightforward manner imply point-sets of size $O\left(n^{3}\right)$. For $k=3,2,1$, we present such universal point-sets of cardinality $O(n), O(n \log n)$, and $O\left(n^{2} / \log n\right)$ respectively.

Theorem 2. For all $n$, there exist universal point-sets of cardinality $O(n)$, $O(n \log n)$, and $O\left(n^{2} / \log n\right)$ that support the drawing of all $n$-vertex planar graphs with at most 3, 2, or 1 bend per edge, respectively.

Table 1. Summary of results - cardinality of universal point-sets for classes of graphs. The first and last results are well-known. All other results are new.

\begin{tabular}{|c|c|c|c|}
\hline Graphs & Number of Points & Number of Bends & Reference \\
\hline outerplanar & $n$ & 0 & {$[8]$} \\
3SP lattice & $n$ & 0 & Thm [1 \\
planar & $O(n)$ & 3 & Thm [2 \\
planar & $O(n \log n)$ & 2 & Thm [2 \\
planar & $O\left(n^{2} / \log n\right)$ & 1 & Thm 2 \\
sub-Hamiltonian & $O(n)$ & 2 & Lemma 3.1 \\
sub-Hamiltonian & $O(n \log n)$ & 1 & Lemma 3.2 \\
planar & $O\left(n^{2}\right)$ & 0 & {$[4,[1]$} \\
\hline
\end{tabular}


Table 1 summarizes our results in terms of which sets of planar graphs can be supported on point-sets of a given cardinality with a specified number of bends.

\section{Preliminaries}

We adopt standard notation from the graph drawing literature and we henceforth assume all graphs have $n$ vertices. Many proofs are omitted for space reasons.

Two of our results rely on point-sets that have a specific form; see, for example, Fig. 2. Two non-intersecting non-linear arcs of curves $\lambda_{1}$ and $\lambda_{2}$ are defined to be biconvex if: each of these arcs is simple and convex, the convex hull of their 4 endpoints completely contains the two arcs, and the line segment joining any point $a$ of $\lambda_{1}$ to any point $b$ of $\lambda_{2}$ does not intersect either arc of curve except at $a$ and $b$; for simplicity, we refer to such arcs of curves as curves. Without loss of generality, we assume the existence of a horizontal line separating the two curves with $\lambda_{1}$ below $\lambda_{2}$. A point-set all of whose points lie on two curves that are biconvex is in biconvex position. We note that point-sets in such a configuration have been used in other contexts under different names.

\section{Universal Point-Sets for Drawing Planar Graphs with Bends}

In this section we establish Theorem 2 by constructing universal point-sets for each of the three cases: 3, 2 or 1 bend per edge allowed. A fundamental tool in our constructions for universal point-sets with bends is the following result proving the existence of a book embedding of planar graphs in which the edges are permitted to cross the spine [6]. A monotone topological book embedding of a planar graph $G$ is a planar drawing such that all vertices of $G$ are represented as distinct points on a spine (i.e. the $x$-axis), and each edge is either represented as an arc in the bottom page (below the $x$-axis), or as an arc in the top page (above the $x$-axis), or as the concatenation of two arcs: the first (leftmost) in the bottom page and the second in the top page with their common crossing point between spine points. See Fig. 1 .

Theorem 3 ([6]). Every planar graph has a proper monotone topological book embedding which can be computed in linear time.
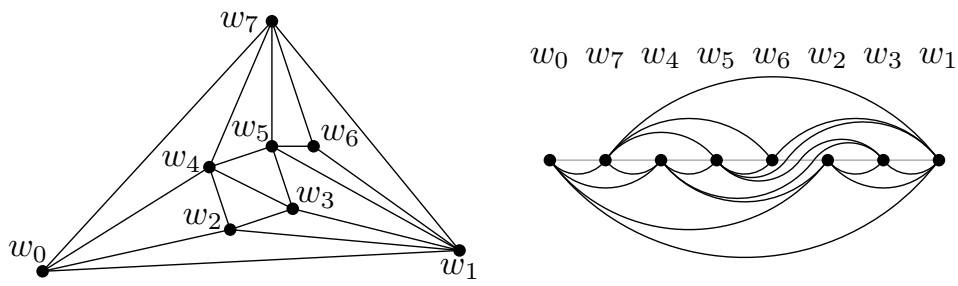

Fig. 1. A graph and a monotone topological book embedding of it 


\subsection{A Set of $\Theta(n)$ Points for Drawing Planar Graphs with Three Bends Per Edge}

Lemma 1. There exists a universal set of $10 n-18$ points that supports the drawing of planar graphs with 3 bends per edge.

Proof. Before introducing the (fixed) universal point-set, we first outline how the graph will be processed. Consider a proper monotone topological book embedding of the input graph (see Fig. 1). For each edge that intersects the spine, introduce a dummy vertex creating an augmented two page book embedding with the vertices of the spine drawn on a horizontal line. There are at most $n+m \leqslant 4 n-6$ vertices on the spine. Imagine a horizontal line slightly above the spine that intersects all arcs in the top page - call these points of intersection from left to right $b_{1}, \ldots, b_{2 a}$ where $a$ is the number of arcs. Note that $a \leqslant 3 n-6$.

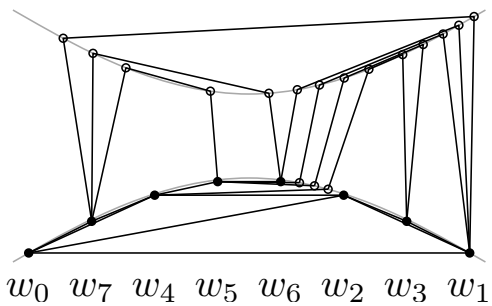

Fig. 2. Example drawing of the graph of Fig. 1 on a biconvex point-set using three bends per edge. The curvature is exaggerated and only the first 14 of the 36 upper curve points and 11 of the 26 lower curve points in the universal point-set are shown.

Consider a point-set that lies on two curves in biconvex position and consists of $6 n-12$ points on the top curve and $4 n-6$ points on the bottom curve. Any such point-set is universal and supports the drawing of all planar graphs with at most three bends per edge. Refer to Fig. 2 for an example of the construction. For any specific graph, its augmented two page book embedding defines the drawing and requires at most $10 n-18$ points. The at most $4 n-6$ vertices on the spine (including dummy vertices) are assigned, in order, to the first points on the bottom curve. The bend-points $b_{1}, \ldots, b_{2 a}$ are assigned to the first $2 a$ points of the upper curve in left to right order and then each arc in the top page is drawn using the associated bend-points. These polylines do not intersect since the upper curve is convex and any segment joining the two curves does not properly intersect these curves. The arcs in the bottom page can be drawn with no bends - they are cords of the bottom curve. Each arc in the top page uses two bend-points. Substituting a bend-point for each of the dummy vertices results in a drawing with at most three bends per edge. 
Note that a sub-Hamiltonian planar graph corresponds exactly to a graph that has a two page (unaugmented) book embedding [6]. Since such graphs do not require dummy vertices, they can be drawn with at most two bends per edge.

\subsection{A Set of $O(n \log n)$ Points for Drawing Planar Graphs with 2 Bends Per Edge}

The geometric idea underlying our construction is as follows. Similar to Section 3.1 we draw the spine vertices of an augmented two page book embedding on a set of points that lie on a slightly concave curve close to the $x$-axis. This implies that all the arcs in the bottom page of the book embedding can be drawn as straight line segments. For arcs in the top page, if the arc is from the $i$ th to the $(i+j)$ th spine vertex, it is drawn to bend at a point at level $j$. We place approximately $n / j$ bend-points approximately equally spaced in the $x$-dimension at level $j$, since only $n / j$ top arcs can have "length" $j$. The bend-point that lies between the $i$ th and $(i+j)$ th spine vertices is used by this arc. Each level is at a $y$-coordinate that is large enough that the drawing of an arc that uses a bend-point at a lower level "nests" inside any drawing of an arc from the same vertex using a higher level bend-point. Of course, for each $j>n / 2$, there can be only one arc of "length" $j$ and it uses a single bend-point at level $j$. The total number of bend-points placed is $O(n \log n)$.

Lemma 2. There exists a universal set of $O(n \log n)$ points that supports the drawing of planar graphs with 2 bends per edge.

\subsection{A Set of $O\left(n^{2} / \log n\right)$ Points for Drawing Planar Graphs with 1 Bend Per Edge}

Lemma 3. There exists a universal set of $O\left(n^{2} / \log n\right)$ points that supports the drawing of planar graphs with 1 bend per edge.

Proof. The construction is similar to that in [7]. We recall briefly this construction, referring to Figures 1 and 3 .

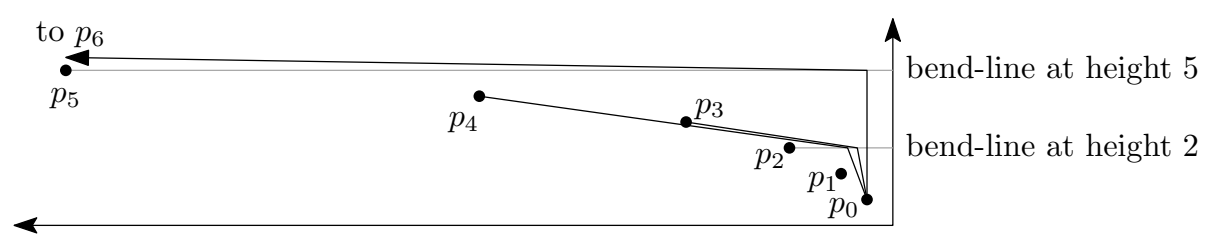

Fig. 3. The one-bend drawing of the three top-page edges adjacent to $v_{0}=w_{1}$ in the graph of Fig. 1 following the construction of [7. The points $p_{6}$ and $p_{7}$ are not shown since the figure is to scale. 
Given a planar graph $G$ with $n$ vertices, we embed the graph on vertices $p_{i}=\left(-2^{i}, i\right)$ for $i=0, \ldots, n-1$ with at most one bend per edge, as follows. We first compute a proper monotone topological book embedding, $\Gamma$ of $G$. We relabel the vertices of that book embedding from right to left, as $v_{0}, \ldots, v_{n-1}$. We then map these vertices to $p_{0}, \ldots, p_{n-1}$, respectively. All the edges below the spine are drawn as straight-line segments. The others are drawn with a bend point as follows. Consider an edge whose rightmost vertex is $v_{i}$ and that intersects the spine on the interval $\left(v_{u}, v_{u+1}\right]$ (inclusive $v_{u+1}$ for the case where the leftmost endpoint of the edge is $\left.v_{u+1}\right)$. Such an edge is drawn with a bend point at the same height as $v_{u}$, and in the vertical strip delimited by $v_{i}$ and $v_{i+1}$. A universal set of points for the bend location can easily be determined in this construction. However, this construction requires a set of size $\Theta\left(n^{3}\right)$ for the bend points since there need to be $n$ bend points on each of $n$ bend lines, and in each of $n-1$ vertical strips (delimited by $v_{i}$ and $v_{i+1}$ ).

This construction can be modified to contain only a subquadratic universal set of points for the bends, while preserving a linear size universal set of points for the vertices. We consider as before a proper monotone topological book embedding, $\Gamma$ of our input graph $G$. We then add on the spine extra isolated dummy vertices so that there is at most one edge crossing the spine between any two spine vertices. Since the number of edges of a planar graph is at most $3 n-6$, we add at most that number of isolated vertices and the total number of vertices is less than $4 n$. Let $G^{\prime}$ be the resulting graph. Note that, if we use the construction of [7] with this augmented graph $G^{\prime}$, there is at most one bend point on each bend line; this yields that, for every bend line, we need only consider one candidate location for the bend points in each vertical strip, leading to a quadratic universal set of points for the bends.

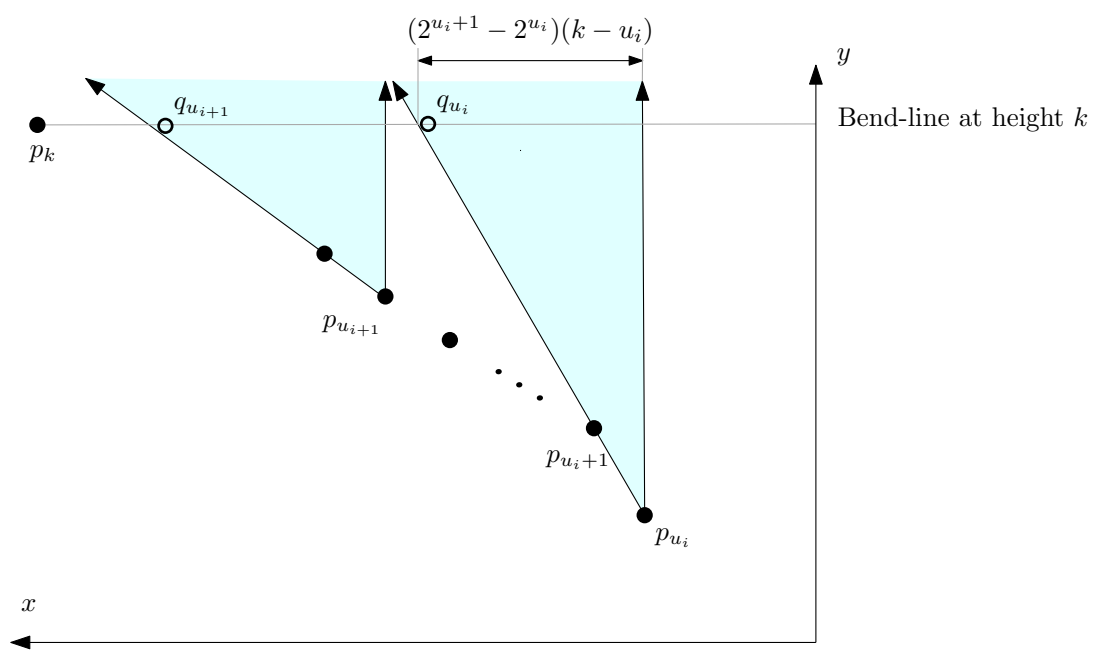

Fig. 4. Placement of bend points on the bend-line at height $k$. The figure is not to scale. 
To obtain a subquadratic size we first construct a set of $4 n$ points $p_{0}, \ldots, p_{4 n-1}$ as in [7] that will support the vertices of any augmented graph $G^{\prime}$. Now on the bend line at height $k$, we place $k / \log k$ candidate vertices for the bend points of the edges that intersect the spine through the window $p_{k} p_{k+1}$. The points on the bend-line at height $k$ are defined as follows. See Fig. 4. The first (rightmost) point $q_{1}$ lies infinitesimally to the right of the line $p_{0} p_{1}$. Let $p_{u_{1}}$ be the rightmost point of $p_{0}, \ldots, p_{4 n-1}$ that is to the left of $q_{1}$. The second point $q_{2}$ is infinitesimally to the right of the line $p_{u_{1}} p_{u_{1}+1}$ (at height $k$ ), and so on.

The addition of dummy points on the spine ensured that there is at most one edge that intersects the spine between two vertices on the spine. Hence, for any given graph, there is at most one bend point used on any bend line. This essentially implies that the construction of [7] works here.

How many bend points are there on a bend-line? It can be shown that there are $\Theta(k / \log k)$ candidate bend points on the bend line at height $k$, and $\Theta\left(n^{2} / \log n\right)$ candidate bend points in total.

\section{Biconvex Point-Sets and Series-Parallel Graphs}

A two terminal series-parallel digraph (also called TTSP-digraph) is a planar digraph recursively defined as follows 512]: A directed edge joining two vertices forms a TTSP-digraph. Let $G^{\prime}$ and $G^{\prime \prime}$ be two TTSP-digraphs; the digraph obtained by identifying the sink of $G^{\prime}$ with the source of $G^{\prime \prime}$ (Series Composition) is also a TTSP-digraph. Let $G^{\prime}$ and $G^{\prime \prime}$ be two TTSP-digraphs; the digraph obtained by identifying the source of $G^{\prime}$ with the source of $G^{\prime \prime}$ and the sink of $G^{\prime}$ with the sink of $G^{\prime \prime}$ (Parallel Composition) is also a TTSP-digraph.

A TTSP-digraph has one source and one sink which are called its poles. Also, a TTSP-digraph is always acyclic and admits a planar embedding with the poles on the same face. A TTSP-digraph is a TTSP lattice if for every edge $(u, v)$, there is no directed path from $u$ to $v$ that does not contain $(u, v)$. Note that a TTSP lattice cannot have multiple edges.

The undirected underlying graph of a TTSP-digraph (resp. lattice) is called a TTSP-graph (resp. TTSP-lattice). We further shorten these terms and refer to them as series-parallel (SP).

Any point-set in general position supports the class of outerplanar graphs 8]. Indeed a point-set in convex position supports exactly the class of outerplanar graphs, and no other planar graphs. Motivated by this insight we now consider the class of planar graphs that are supported by a point-set in which $n / 2$ points are on one convex curve and the remainder are on another convex curve - in biconvex position. Clearly outerplanar graphs can be supported by this point-set and efficient algorithms such as that developed by Bose 1 exist. We show that any $(n / 2, n / 2)$ biconvex point-set is universal for a subclass of the series-parallel graphs. Since our purpose is to exhibit universal point-sets for classes of planar graphs, the balancing condition is critical and since the number of vertices could be odd, the balancing must allow for one vertex to be placed arbitrarily. 
A planar graph $G$ is biconvex if there exists a crossing-free straight-line drawing $\Gamma$ of $G$ with all vertices located on the curves $\lambda_{1}$ and $\lambda_{2}$. A planar graph $G$ is balanced biconvex if it is biconvex with a drawing $\Gamma$ in which the numbers of vertices on the two curves differ by at most one; more formally if:

for $n$ even, $n / 2$ vertices are on $\lambda_{1}$ and $n / 2$ vertices on $\lambda_{2}$ (called uniform and denoted as $\Gamma^{=}$), and for $n$ odd, either:

- $\frac{n-1}{2}$ vertices are on $\lambda_{1}$ and $\frac{n+1}{2}$ vertices are on $\lambda_{2}$ (called top-heavy and denoted as $\Gamma^{+}$) or

- $\frac{n+1}{2}$ vertices are on $\lambda_{1}$ and $\frac{n-1}{2}$ vertices are on $\lambda_{2}$ (called bottom-heavy and denoted as $\Gamma_{+}$)

Our construction is recursive and attempts to contain the drawing of the SP lattice in a box spanning the biconvex curves with a balanced number of vertices on each curve and with $s$ and $t$ forming a diagonal of the box. Unfortunately, such a strong invariant cannot be maintained and slightly weaker conditions must be carefully considered.

A series-parallel digraph with poles $s$ and $t$ is bottom-cornered if it is balanced biconvex with a drawing $\Gamma$ ( $n$ even) or $\Gamma_{+}$(n odd) such that:

1. there exists a box (i.e. a convex quadrilateral) $B(s, t)$ with $s$ on $\lambda_{1}$ and $t$ on $\lambda_{2}, \overline{s t}$ forms one diagonal of $B$, and the other diagonal has one corner on $\lambda_{1}$ and one on $\lambda_{2}$, and

2. the entire drawing lies inside $B$.

Similarly, a series-parallel digraph with poles $s$ and $t$ is top-cornered if it is balanced biconvex with a drawing $\Gamma$ ( $n$ even), or $\Gamma^{+}(n$ odd $)$ such that conditions 1 and 2 hold. If a series-parallel graph is both top-cornered and bottom-cornered, it is called double-cornered - i.e. if $n$ is odd, there exist two drawings $\Gamma^{+}$and $\Gamma_{+}$both of which satisfy conditions 1 and 2 .

In some situations, only weaker conditions on the drawings can be maintained, in which one of $t$ or $s$ is contained strictly inside a box rather than on the diagonal forming the box:

$1^{\prime}$. there exists a box $B(s, x)$ with $s$ on $\lambda_{1}$ and $x$ on $\lambda_{2}, \overline{s x}$ forms one diagonal of $B(s, x)$, and the other diagonal has one corner on $\lambda_{1}$ and one on $\lambda_{2}$ and $t$ is on $\lambda_{2}$ inside $B(s, x)$.

$1^{\prime \prime}$. there exists a box $B(x, t)$ with $x$ on $\lambda_{1}$ and $t$ on $\lambda_{2}, \overline{x t}$ forms one diagonal of $B(x, t)$, and the other diagonal has one corner on $\lambda_{1}$ and one on $\lambda_{2}$ and $s$ is on $\lambda_{1}$ inside $B(x, t)$.

A series-parallel graph with source $s$ and $\operatorname{sink} t$ is bottom half-cornered if it is balanced biconvex and conditions 1' and 2 hold; similarly, if conditions 1 " and 2 hold, then the graph is top half-cornered.

In the lemmas that follow, we demonstrate only the existence of biconvex drawings for the graphs that we consider. The following lemma indicates that this is sufficient to claim a universal biconvex point-set of suitable size. 
Lemma 4. If a graph $G$ on $n$ vertices has a balanced biconvex drawing, then every balanced biconvex point-set of size $n$ supports $G$.

A series-parallel graph in which every vertex is of maximum degree 3 is denoted as 3SP. We distinguish between two critical cases. If both the source and sink of a 3SP lattice have degree $\leqslant 2$ then the graph is called thin and otherwise (i.e. if either pole has degree 3) it is called thick. It is the class of 3SP lattices that we show to be balanced biconvex. There are several cases to consider depending on whether the graph is biconnected or not, and whether the graph is thin or thick. Our proof is recursive in nature - interior components are replaced by appropriate balanced boxes. Lemmas 5-14 distinguish and organize these cases and Fig. 5 provides a simple example of each case, the type of drawing obtained, and the prerequisite lemmas used in the proof.

Lemma 5. A simple path consisting of $n \geqslant 2$ vertices from $s$ to $t$ is doublecornered.

First we present the lemmas used for subcases that are thin: biconnected and then not biconnected.

Lemma 6. Let $G$ be a biconnected thin $3 S P$ lattice. Then $G$ is double-cornered.

Lemma 7. Let $G$ be a thin 3SP lattice with source $s$ and sink $t$. If either $\operatorname{deg}(s)=\operatorname{deg}(t)=1$ or $\operatorname{deg}(s)=\operatorname{deg}(t)=2$ then $G$ is double-cornered.

Lemma 8. Let $G$ be a thin $3 S P$ lattice with source $s$ and sink $t$. If $\operatorname{deg}(s)=1$ and $\operatorname{deg}(t)=2$ then $G$ is bottom-cornered.

Lemma 9. Let $G$ be a thin $3 S P$ lattice with source $s$ and sink $t$. If $\operatorname{deg}(s)=2$ and $\operatorname{deg}(t)=1$ then $G$ is top-cornered.

The next sequence of lemmas pertains to the cases when the global poles have degree 3. The drawings obtained rely on the previous lemmas and are balanced biconvex, but may not be double-cornered.

Lemma 10. Let $G$ be a biconnected $3 S P$ lattice with source $s$ and sink $t$. If $\operatorname{deg}(s)=\operatorname{deg}(t)=3$ and $G$ consists of 3 series components combined in parallel, then $G$ is balanced biconvex.

Lemma 11. Let $G$ be a biconnected thick $3 S P$ lattice with source $s$ and sink $t$. If $\operatorname{deg}(s)=\operatorname{deg}(t)=3$ then $G$ is balanced biconvex.

Lemma 12. Let $G$ be a biconnected thick $3 S P$ lattice with source $s$ and sink $t$. If $\operatorname{deg}(s)=2$ and $\operatorname{deg}(t)=3$ then $G$ is bottom half-cornered.

Lemma 13. Let $G$ be a biconnected thick $3 S P$ lattice with source $s$ and sink $t$. If $\operatorname{deg}(s)=3$ and $\operatorname{deg}(t)=2$ then $G$ is top half-cornered.

In the final case, $G$ is not biconnected and at least one of the global poles has degree 3 . 


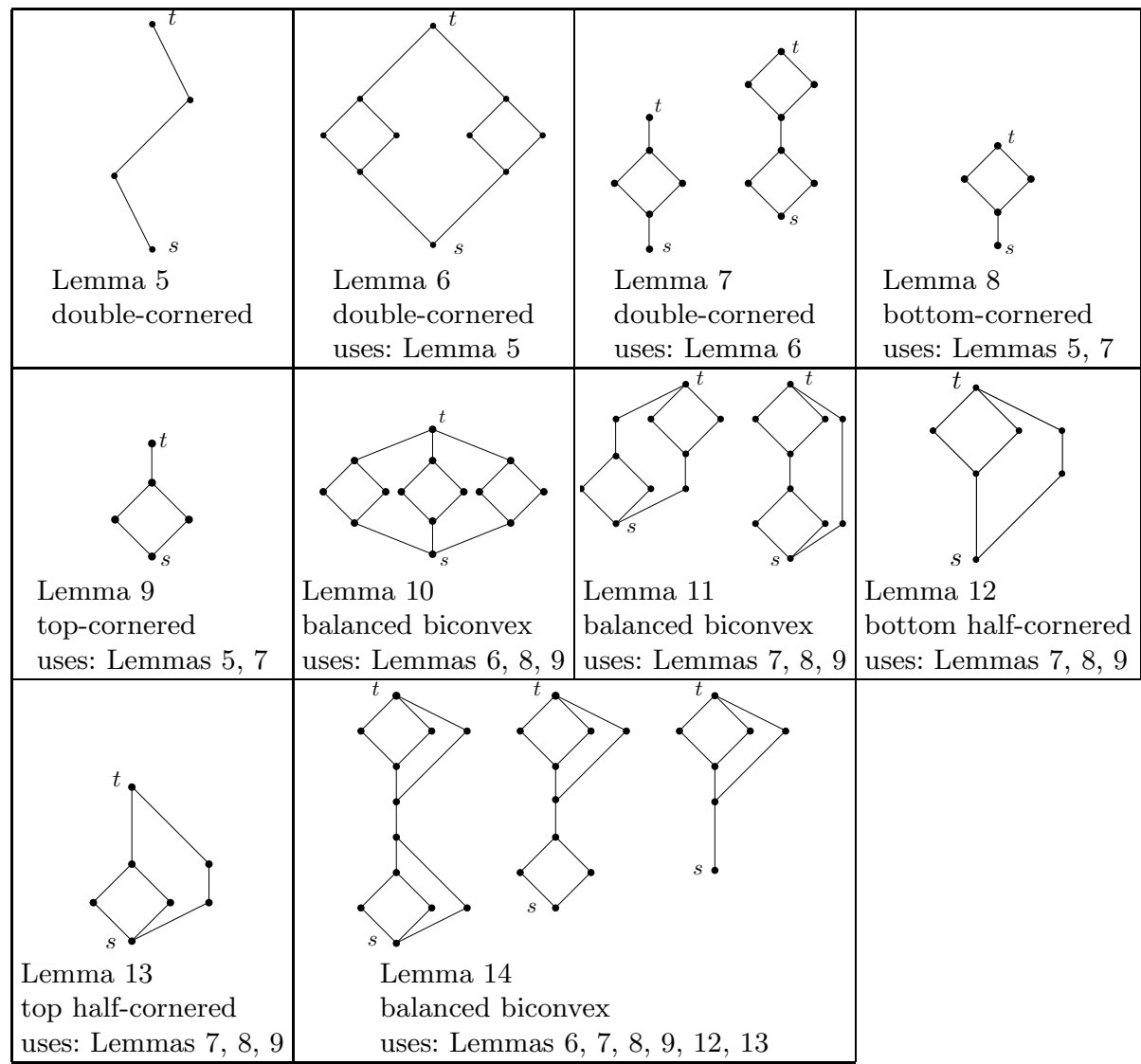

Fig. 5. Roadmap of the various cases

Lemma 14. Let $G$ be a thick $3 S P$ lattice with source $s$ and sink $t$. If $\operatorname{deg}(s)=3$ or deg $(t)=3$ then $G$ is balanced biconvex.

Biconvex point-sets are the only known point-sets of size $n$ that universally support some class of planar graphs other than the outerplanar graphs.

\section{Conclusions and Open Problems}

Our main contributions in this paper are stated in Theorems 1 and 2: that any balanced biconvex point-set supports the straight-line drawing of any 3SP lattice, and the specification of universal point-sets for drawings of any planar graph with a small number of bends per edge.

Closing the gap between the upper and lower bounds of the cardinality of a universal point-set for planar graphs with no bends allowed remains an open problem. When $k$ bends per edge are permitted, universal point-sets of smaller asymptotic cardinality may be determined for $k=1,2$. 


\section{References}

1. Bose, P.: On embedding an outer-planar graph on a point set. Computational Geometry: Theory and Applications 23, 303-312 (2002)

2. Braß, P., Cenek, E., Duncan, C.A., Efrat, A., Erten, C., Ismailescu, D., Kobourov, S.G., Lubiw, A., Mitchell, J.S.B.: On simultaneous planar graph embeddings. Comput. Geom. 36(2), 117-130 (2007)

3. Chrobak, M., Karloff, H.: A lower bound on the size of universal sets for planar graphs. SIGACT News 20(4), 83-86 (1989)

4. de Fraysseix, H., Pach, J., Pollack, R.: How to draw a planar graph on a grid. Combinatorica 10, 41-51 (1990)

5. Di Battista, G., Eades, P., Tamassia, R., Tollis, I.G.: Graph Drawing. Prentice-Hall, NJ (1999)

6. Di Giacomo, E., Didimo, W., Liotta, G., Wismath, S.K.: Curve-constrained drawings of planar graphs. Computational Geometry 30, 1-23 (2005)

7. Everett, H., Lazard, S., Liotta, G., Wismath, S.: Universal sets of $n$ points for one-bend drawings of planar graphs with $n$ vertices. Discrete and Computational Geometry 43(2), 272-288 (2010)

8. Gritzmann, P., Mohar, B., Pach, J., Pollack, R.: Embedding a planar triangulation with vertices at specified points. Amer. Math. Monthly 98(2), 165-166 (1991)

9. Kaufmann, M., Wiese, R.: Embedding vertices at points: Few bends suffice for planar graphs. Journal of Graph Algorithms and Applications 6(1), 115-129 (2002)

10. Kurowski, M.: A 1.235 lower bound on the number of points needed to draw all n-vertex planar graphs. Inf. Process. Lett. 92(2), 95-98 (2004)

11. Schnyder, W.: Embedding planar graphs on the grid. In: Proc. 1st ACM-SIAM Sympos. Discrete Algorithms (SODA 1990), pp. 138-148 (1990)

12. Valdes, J., Tarjan, R.E., Lawler, E.L.: The recognition of series-parallel digraphs. SIAM J. Comput. 11(2), 298-313 (1982) 